\title{
Biodegradable versus titanium plates and screws for paediatric facial skeleton fractures
}

\author{
Janickova $\mathrm{M}^{1}$, Statelova $\mathrm{D}^{1}$, Mikuskova $\mathrm{K}^{1}$, Jesenak $\mathrm{M}^{2}$, Malachovsky $\mathrm{I}^{1}$ \\ Department of Stomatology and Maxillofacial Surgery, Jessenius Faculty of Medicine in Martin, Comenius \\ University in Bratislava, University Hospital in Martin, Slovakia. malachovsky@unm.sk
}

\begin{abstract}
OBJECTIVE: The use of biodegradable materials represents a new option in the treatment of paediatric facial skeleton fractures. The benefits of a resorbable system include reductions in time for long-term stability, diminished immobilisation period, and elimination of painful procedures for implant removal. The resorption of the material did not influence bone repair and growth. Bioresorbable plates and screws get completely excreted through physiological routes.

MATERIAL AND METHODS: The age of the patients ranges from that of pre-schoolers till 18 years. The mean age of boys and girls was 12.18 (range 4-18 years) and 13.59 (range 5-18 years), respectively.

RESULTS: During the ten-year period, 168 children and adolescents, 136 boys and 32 girls, were treated. The conservative treatment was performed in 67 patients (39.9\%). Open surgical reduction was performed in 101 patients (60.1\%). Bioresorbable plates were applied in 44 patients (43.6 \% of surgically treated).

CONCLUSION: The implementation of biodegradable osteosynthetic materials is optimal for the treatment of fractures of the middle third of the facial skeleton, lower part of the upper third of the facial skeleton, mandibular body and parasymphysis (Tab. 3, Fig. 3, Ref. 31). Text in PDF www.elis.sk.

KEY WORDS: children, maxillofacial fractures, bioresorbable osteosynthesis, titanium osteosynthesis.
\end{abstract}

\section{Introduction}

The aim of the present study was to analyse the use of titanium plates and screws versus biodegradable plates and screws in pursuit of fixating the facial skeleton fractures in children. There is still no consensus as to which material is best suited for repairing maxillofacial fractures. Paediatric facial fracture management is critical to optimal long-term success (6).

Internal fixation implies an approach with subsequent subperiosteal dissection, which interrupts the osteogenic potential of the periosteum and creates scarring, which may further restrict growth.

Titanium microplates and miniplates offer improved initial stability but its application in children is limited because of con-

${ }^{1}$ Department of Stomatology and Maxillofacial Surgery, Jessenius Faculty of Medicine in Martin, Comenius University in Bratislava, University Hospital in Martin, Slovakia, and ${ }^{2}$ Department of Paediatrics, Jessenius Faculty of Medicine in Martin, Comenius University in Bratislava, University Hospital in Martin, Slovakia

Address for correspondence: I. Malachovský, MD, PhD, Department of Stomatology and Maxillofacial Surgery, Jessenius Faculty of Medicine in Martin, Comenius University in Bratislava, University Hospital in Martin, Kollarova 2, SK-036 59 Martin, Slovakia.

Phone: +421.43.4203-357

Acknowledgement: This study was supported by Grant of the Ministry of Health of the Slovak Republic 2007/49-MFN-02 (Indication criteria and optimal therapeutic methods in treatment of children with fractures in the maxillofacial region by use of biodegradable osteosynthesis materials and comparison to standard methods), as well as by the project Center of Experimental and Clinical Respirology, which was co-funded by EU sources. cerns over growth restrictions, stress shielding, corrosion and palpability. The disadvantages of titanium plates and screws also include implant migration, extrusion, thermal sensitivity and the need for secondary removal surgery $(25,26)$. In contrast, the main advantage of internal resorbable fixation is the gradual transfer of load to the healing bone during resorption, elimination of any secondary operations for implant removal, as well as no interference when acquiring CT or MRI $(3,5,16,18)$. There have been some reports of using biodegradable plates and screws for the fixation with focus on associated infection, swelling and foreign body reaction. However, infection and foreign body reaction can occur also in patients with titanium plates and screws.

The use of biodegradable plates and screws is a well-known alternative to titanium in the osteosynthesis of maxillofacial fractures. In recent decades, the use of biodegradable plates and screws in the midface and craniofacial area for the treatment of mandibular fractures and in orthognathic surgery has been described in the literature (23, 30, 32, 33). The commonly used biodegradable plating systems are mainly composed of polyglycolic acid (PGA), poly L-lactic acid and their copolymers. Generally, the plate bending requires a heating device to cause the polymer chains to bend and not break, while placing a resorbable screw requires pre-tapping of the screw threads before inserting the screw. It has been reported that paediatric facial fractures have been treated with ultrasound-aided fixation of biodegradable implants (1).

Maxillofacial trauma in a paediatric group of patients is particularly challenging, as we observe that the facial skeleton in these patients significantly differs from that of adults (14). These 


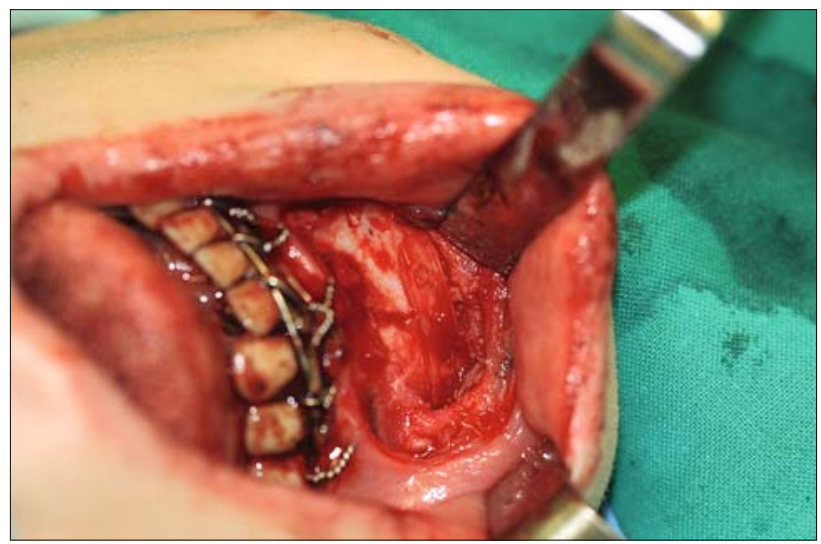

Fig. 1. Clinical photograph of a paediatric patient whose mandible fracture was stabilized with linear $2.0 \mathrm{~mm}$ resorbable plate and screws.

differences include the growth potential, small size of the bones, small volume of the paranasal sinuses, presence of the tooth germs in the jaws during primary and mixed phases of dentition, rapid healing process and frequently difficult compliance and cooperation when compared to those in adults $(4,12,20,28)$.

The incidence of paediatric facial fractures is low (ranges from approximately $1 \%$ in children under the age of five years up to $8 \%$ in children younger than 12 years), most likely owing to the flexibility of the facial bone, lack of pneumatisation of the paranasal sinuses and protection of the malar region by the prominent buccal fat pad in infants (26). Pertaining to the face, the retruded position of the midface in the child relative to the protecting skull and prominent mandible is an important reason for the lower incidence of midface fractures in paediatric patients (22).

Maxilla grows downward and forward, which is allowed by the ideally situated sutures attaching the maxilla posteriorly and superiorly and by growth of the cranial base behind it $(7,31)$. Furthermore, young children are less often involved in occupational or violence-related accidents, which are typical features of adult facial fractures. The phenomenal increase in traffic density has led to a tremendous rise in the number of traffic accidents leading to facial injuries, while children are the most unfortunate victims $(19,26)$. Interpersonal violence is a relatively infrequent cause of injury to the craniofacial skeleton in the paediatric population (13).

Because of the unique anatomy, dentition and growth of the paediatric patient, the management of paediatric facial fractures requires true diligence with a variance in treatment ranging from soft diet to open reduction and internal fixation (10).

The conservative approach to the treatment of maxillofacial trauma in children was common for many reasons. The presence of tooth buds and elasticity of the paediatric bone were the factors that established splinting and intermaxillary fixation as a standard treatment of mandibular fractures in children during the phase of deciduous dentition $(8,29,33)$.

When discussing the complications and adverse outcomes related to facial fractures, some authors have defined three types of adverse outcomes that should be considered, namely those intrinsic to, or concomitant with the fracture itself as e.g. the loss of

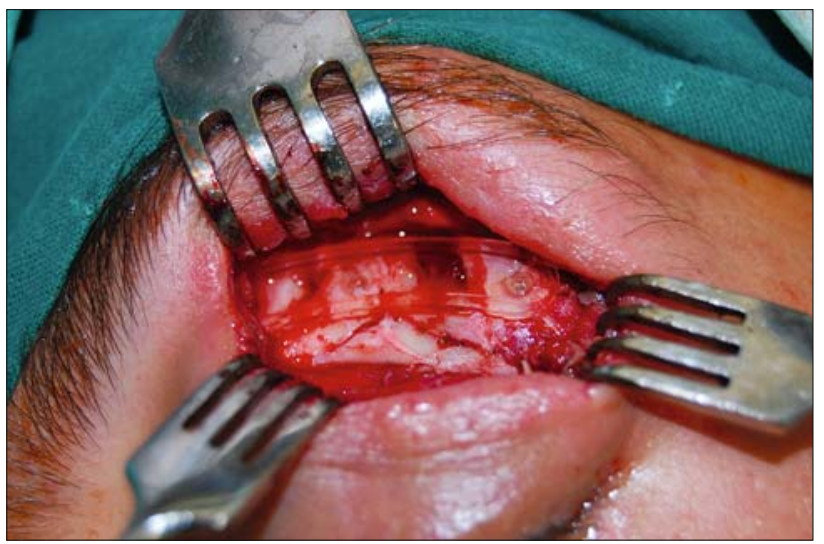

Fig. 2. Clinical photograph of a paediatric patient whose frontal bone fracture was reconstructed with linear $2.0 \mathrm{~mm}$ resorbable plate and screws.

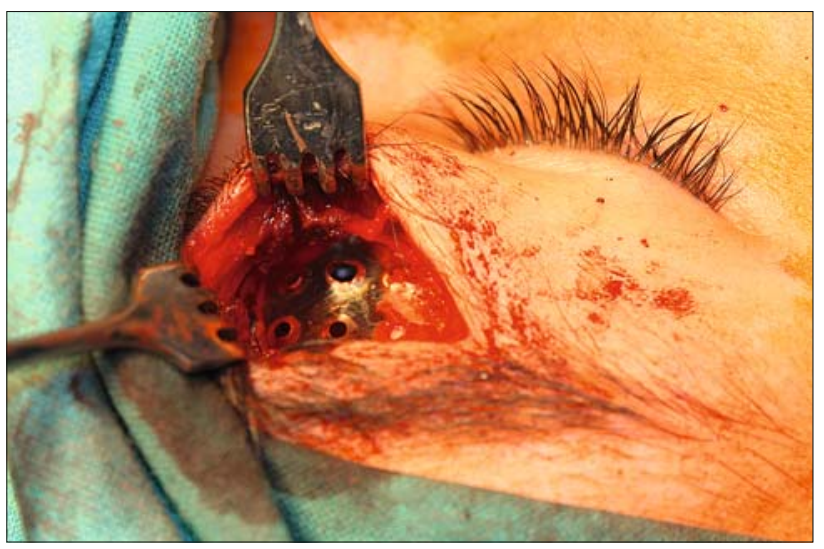

Fig. 3. Clinical photograph of a paediatric patient whose frontal bone fracture was stabilized with resorbable mesh and screws.

a permanent tooth (type 1), those secondary to intervention and surgical management as e.g. marginal mandibular nerve palsy (type 2), and those resulting from subsequent growth and development such as asymmetric growth (type 3) (15).

At the Department of Stomatology and Maxillofacial Surgery of the Jessenius Faculty of Medicine in Martin of Comenius University in Bratislava and University Hospital in Martin we were among the first ones in Slovakia to start using bioresorbable materials. We have been using it successfully since 2007 in frame of a grant of the Ministry of Health of the Slovak Republic. In our retrospective study, we aimed to analyse and assess the treatment methods and outcomes of maxillofacial fractures in children and young adolescents during the last ten years. We focused on the use of biodegradable materials and compared this approach with conventional therapeutic strategies.

\section{Materials and methods}

The records of all paediatric patients with fractures of the facial skeleton, who were treated at the Department of Stomatology and Maxillofacial Surgery of the Jessenius Faculty of Medicine in 
Martin of Comenius University in Bratislava and University Hospital in Martin from January 2007 to February 2017 were reviewed retrospectively. The age of patients included in the study ranged from that of pre-schoolers to 18 years. The mean age of boys and girls was 12.18 (range 4-18 years) and 13.59 (range 5-18 years), respectively. During the 10 -year period, 168 children and adolescents, 136 boys and 32 girls, were treated. We observed fractures of the lower part of the upper third of the facial skeleton, medial third of the facial skeleton and mandible. The information extracted from each patient's medical records included gender, age, aetiology, fracture type, site and treatment performed.

Fractures of the maxilla and midface were classified according to the Le Fort system, and those of the mandibular and zygomatic complex were classified according to Killey (26). The present study identified optimum therapeutic treatments in children with fractures of the maxillofacial skeleton.

Conservative treatment observation and closed reduction was mainly applied in cases of non-displaced or minimally displaced fractures (e.g. condylar and dentoalveolar fractures). The surgical approach was strictly intraoral in cases of lower jaw fractures. The approach was either through the eyebrow and lower eyelid for zygomatic complex fractures or through existing facial lacerations in fractures of the middle and upper of the face. As for the internal fixation of bones fragments, we used bioresorbable osteosynthetic materials $(1.5 \mathrm{~mm}$ and $2 \mathrm{~mm}$ resorbable plates and screws) and titanium plates and screws. We exclusively used resorbable plates, co-polymer plates and screws composed of polylactic and polyglycolic acids (PLLA-PGA), $82 \%$ and $18 \%$, respectively. The combination of PLLA and PGA and the ratio of the materials allow to maintain almost $75 \%$ of its strength in the initial 3 months.

Tab. 1. Mechanism of injury.

\begin{tabular}{lcc}
\hline Mechanism of Injury & Total & \% of patients \\
\hline Bicycle accidents & 50 & 29.76 \\
Common falls & 33 & 19.64 \\
Sports accidents & 31 & 18.45 \\
Road traffic accident & 30 & 17.86 \\
Assault & 21 & 12.50 \\
Other & 3 & 1.79 \\
\hline Total & 168 & 100 \\
\hline
\end{tabular}

Data are numbers and percentage of patients
The follow-up period was at least one year for all patients, and it was extended to the end of skeletal growth, depending on case severity.

\section{Results}

During the analysed period, altogether 168 children and adolescents (136 boys, $81 \%$ ) were treated. The type and site of fractures were registered according to involved bones as follows: mandible (body, condyles, dentoalveolar, angle, symphysis and parasymphysis), midface (Le Fort, zygomatic complex, blow-out, nasoethmoidal and frontal bones). The 168 children treated had 180 fracture sites. A total of 156 children (92.9\%) had a single fracture, and 12 children (7.1\%) had multiple fractures. We observed that most frequently occurring fracture was that of the mandible (70 cases; $41.7 \%$ ). The zygomatic complex was affected in 54 cases (32.1\%), the maxilla was affected in 21 cases (12.5\%), the orbital walls (blow-out fractures) were affected in 13 cases (7.7 \%), the frontal bone was affected in 13 cases $(7.7 \%)$, and the nasoethmoidal was affected in two cases (1.2\%). Most common were mandibular fractures that included parasymphysis with condylar fractures, followed by angle and body fractures. There were 48 casualties (68.6\%) presented with simple mandibular fractures; 17 (24.3\%) patients presented with double mandibular fractures, and five (7.1\%) patients had multiple mandibular fractures. Table 1 shows the causes of injury.

Conservative treatment and close reduction were common methods of treating mandibular fractures. These treatments included liquid diet, intermaxillary fixation (IMF rigid or elastic for a shortened time of about two weeks due to the fracture type) followed by active physiotherapy in 41 cases (58.6 \%). All condylar fractures were treated conservatively in children younger than 16 years (for the purpose of preventing damage to the growth zone). The conventional osteosynthetic material was used in cases where the treatment aimed at repairing mandibular fracture biomechanics while surgical reduction and fixation were required. Titanium mini- or microplates and screws were used in 20 fractures sites (28.6\%), followed by resorbable plates used in 9 of the total of 70 mandibular fracture sites (12.9\%). The distribution of osteosynthesis plates at the fracture sites is shown in Table 2.

Zygomatic complex fractures were the most frequent fractures of the midface. Fifty-four of the total of 168 cases in this group

Tab. 2. Distribution of osteosynthesis material at fractures sites and treatment methods.

\begin{tabular}{|c|c|c|c|c|}
\hline Fracture site & Conservative treatment & Resorbable plates & Titanium plates & Total; \% of total fractures \\
\hline Mandible & 41 & 9 & 20 & $70(38.9 \%)$ \\
\hline Zygomatic complex & 18 & 15 & 21 & $54(30.0 \%)$ \\
\hline Maxilla & 0 & 11 & 10 & $21(11.7 \%)$ \\
\hline Frontal Bone & 2 & 4 & 7 & $13(7.2 \%)$ \\
\hline Blow-out & 0 & 9 & 4 & $13(7.2 \%)$ \\
\hline Proc. alveolaris max. & 7 & 0 & 0 & $7(3.9 \%)$ \\
\hline Nasoethmoidal & 0 & 1 & 1 & $2(1.1 \%)$ \\
\hline$\%$ & 37.78 & 27.22 & 35.00 & 100 \\
\hline
\end{tabular}

Data are numbers of patients and percentage of fractures

Proc. alveolaris max. - processus alveolaris maxillae 
Tab. 3. Complications related to plates and screws and numbers of patients who underwent secondary surgery for plate removal.

\begin{tabular}{lcc}
\hline & Resorbable plates & Titanium plates \\
\hline Infection, $\mathrm{n}(\%)$ & $1(2.04)$ & 0 \\
Extrusion, $\mathrm{n}(\%)$ & 0 & $1(1.59)$ \\
Palpability, n (\%) & 0 & $5(7.93)$ \\
Malocclusion, n & 0 & 0 \\
Malunion, non-union, n & 0 & 0 \\
Secondary surgery, n (\%) & 0 & $8(12.70)$ \\
\hline
\end{tabular}

Data are numbers of patients and percentage of patients with an appropriate treatment method

(32.1\%) suffered fractures of the ZMC (zygomatic complex). The indicated treatment for this type of fracture type, comminution or dislocation of the fragments was osteosynthesis with titanium plates and screws in 21 cases (38.9\%). The sufficiently applied treatment was conservative treatment (18 cases; $33.3 \%$; observation only or elevation with a bone hook). Fifteen cases (27.8 \%) were operated using bioresorbable plating system.

Fractures of the maxilla (LeFort and alveolar process) were present in 21 of the total 168 cases (12.5\%). Resorbable plates were applied for the treatment of these fractures in eleven cases (52.4\%), and the titanium plates system was used in ten cases (47.6 \%). Closed alveolar process fracture reduction and immobilization with arch bars was performed in seven cases (4.2\%).

Frontal bone fractures (mostly supraorbital region) constituted 13 of the total of 168 cases ( $7.7 \%$ ). In seven of these cases (53.8 $\%)$, osteosynthesis with titanium plates and screws was indicated. The resorbable system was used in four cases (30.8 \%), whereas two cases (15.4\%) were managed conservatively.

Blow-out fractures were treated through the lower eyelid incision with a careful release of entrapped soft tissues while the reconstruction of the orbital floor defects using alloplastic resorbable material was done in nine cases (69.2 \%) and titanium mesh in four cases (30.8\%).

Our method of fixating the nasoethmoidal fractures (NOE) was done with resorbable plates and screws in one of NOE cases ( $50 \%$ ), and the titanium system was used in one of NOE cases (50\%). Table 3 demonstrates observed complications. A plate-related infection was observed in one patient with biodegradable plates and screws. Five patients complained of the palpability of titanium plates and screws. The extrusion of titanium plates and screws was observed in one patient. Eight patients with titanium plates and screws underwent the plate removal surgery.

\section{Discussion}

Conservative treatment is the most reliable approach in the management of maxillofacial injuries in children. The criteria for choosing the conservative treatment include no or minimal displacement of the fractures, reproducibility of adequate occlusion or minimal discrepancy in occlusion, and no facial disfigurement. The conservative treatment was reported in literature to range between $30 \%$ and $75 \%$ depending on the country $(19,26)$. In consistency with the published data, we conclude that the conservative approach was the most common treatment method of paediatric facial skeleton fractures in our patient group.

Mandibular fractures account for one third of facial fractures in children and are more often treated conservatively compared to adults. The primary growth centre for the mandible is situated in the condyle and an injury to this area can result in growth disturbance and temporomandibular joint ankylosis. On the other hand, the treatment of condylar head fracture in adults is different. Despite the controversy in the management of condylar head fractures, it is generally agreed that open reduction and internal fixation especially in cases of condylar head fractures, namely in types B and C of Neff 's classification, provides good functional results (11).

Resorbable system for paediatric patients enables fast and solid bone healing during the immobilization period (33). Tooth bud injury is unlikely because the drill hole and tapping of the screw threads penetrate only the outer cortex. Subsequent resorption of the screws removes any potential obstruction to tooth eruption $(21,24)$.

The use of resorbable plates in association with dental arch stabilization can provide good stabilization for mandibular fractures and is a promising approach in the treatment of displaced mandibular fractures of ramus, body, parasymphysis and symphysis in children $(34,27)$. Based on our study, we confirm that the bioresorbable plate system provides a reliable stability for osteosynthesis in cases of fractures of the mandibular body, parasymphysis and symphysis.

Iatrou et al (16) reported 156 children during a 9-year period with 208 sites of facial fractures. Open surgical reduction was performed in $66.8 \%$, and closed reduction was performed in $33.2 \%$ of the reported cases. Titanium mini- and microplates were applied in $71.4 \%$, and bioresorbable plates were used in $28.6 \%$. IMF was applied in 36 cases. Their experience suggests that biodegradable plates and screws offer a satisfactory solution for osteosynthesis, and these materials offer significant advantages in cases of frontal and zygomatic fractures as described $(12,16,28)$. Our study shows that biodegradable plating systems are effective for treating midfacial fractures.

Singhal et al (26) reported 110 children with facial fractures during a 45-month period. Paediatric maxillofacial fractures were treated with conservative observation alone in $88 \%$ of cases, close reduction in $16.3 \%$ and open reduction in only $5.4 \%$ cases. All patients with condylar fractures were managed conservatively. The fractures, which were displaced and had severe occlusal disturbances, were operated using bioresorbable plating system.

Bell et al (2) reported interesting observations relating to the differences in complication rates (infection, malunion, nonunion, enopthalmos, ectropion and entropion) between titanium and bioresorbable groups. The two groups were not equivalent. Fourteen of the 16 complications occurred in 222 patients with titanium fixation, and only two of the 16 complications occurred in 59 patients with biodegradable fixation. These findings suggest a lower complication rate with biodegradable fixation than with titanium (9).

Jingang et al (17) fixed 42 fractures with 42 resorbable plates. Two patients developed small fistulas at the internal incision 2 months after surgery; the fistulas healed after 1 month without spe- 
cial treatment. In the other patients, the incision healed well, there was facial symmetry, the mouth opening was $35 \mathrm{~mm}$ and occlusion was good. They describe no adverse effect on fracture healing.

\section{Conclusion}

The ideal method of management of maxillofacial fractures in children is that which is the simplest and produces an adequate reduction and stabilization.

The most important benefits are that these materials do not cause growth disturbances of the facial skeleton and they do not require removal, which thus reduces iatrogenic soft tissue disruption.

Resorbable plates are composed of alpha-hydroxy acid polymer which breaks down through hydrolyzation and their end products are carbon dioxide and water.

One significant advantage of resorbable screws in paediatric fracture is the avoidance of potential odontogenic injury.

We applied the atraumatic bioresorbable osteosynthetic materials in cases where surgical treatment was required according to the type of fracture, comminution or dislocation of the fragments while respecting the growth and further development of the facial skeleton.

Our experience suggests that the implementation of biodegradable osteosynthetic materials is optimal for the treatment of fractures of the middle third of the facial skeleton, lower part of the upper third of the facial skeleton, mandibular body and parasymphysis.

The conventional titanium material has proved to be particularly effective in the treatment of dislocated or comminuted fractures of mandible, mainly in the region of mandibular angle.

Stability and firmness of the applied bioresorbable material in the observed cases was highly sufficient. We did not record tissue intolerance (except for one case of inflammation), growth defects, failure of osteosynthesis or disturbances in occlusion.

In terms of cost, the pricing of resorbable plates and screws is comparable to titanium plates and screws in most situations.

According to our experiences these bioresorbable materials are beneficial in the treatment of childhood fractures of maxillofacial skeleton.

However, further clinical studies are needed to assess the long-term results of facial fractures treatment with biodegradable systems.

\section{References}

1. Aldana PR, Wieder K, Postlethwait RA, James HE, Steinberg B. Ultrasound aided fixation of biodegradable implants in pediatric craniofacial surgery. Pediatr Neurosurg 2011; 47: 349-353.

2. Bell RB, Kindsfater CS. The use of biodegradable plates and screws to stabilize facial fractures. J Oral Maxillofac Surg 2006; 64: 31-39.

3. Bergsma JE, de Bruijn WC, Rozema FR, Bos RR, Boering G. Late degradation tissue response to poly (L-lactid) bone plates and screws. Biomaterials 1995; 16: 25-31.

4. Böstman O, Hirvensalo E, Mäkinen J, Rokkanen P. Foreing body reactions to fracture fixation implants of biodegradable synthetic polymers. J Bone Joint Surg (Br) 1990; 72: 592-596.
5. Cole P, Kaufman Y, Hollier LH. Managing the Pediatric Facial Fractures. Craniomaxillofac Trauma Reconstr 2009; 2 (2): 77-83.

6. Dostalova T, Eliasova H, Gabcova D, Feberova J, Kaminek M. Contemporary skull development-palatal angle analysis. Bratisl Med J 2015; 116 (3): 143-146.

7. Eppley BL, Sadove AM, Havlik RJ. Resorbable plate fixation in pediatric craniofacial surgery. Plast Reconstr Surg 1997; 100: 352-355.

8. Gerber B, Kiwanuka P, Dhariwal D. Orbital Fractures in children: A review of outcomes. Brit J Oral Maxillofac Surg 2013; 51: 789-793.

9. Goth S, Sawatari Y, Peleg M. Management of Pediatric Mandible Fractures. J Craniofac Surg 2012; 23 (1): 47-56.

10. Hirjak D, Machon V, Beno M, Galis B, Kupcova I. Surgical treatment of condylar head fractures, the way to minimize the posttraumatic TMJ ankyloses. Bratisl Med J 2017; 118 (1): 17-22.

11. Holland AJ, Broome C, Stienberg A, Case DT. Facial fractures in children. Pediatr Emerg Care 2001; 17: 157.

12. Hoppe IC, Kordahi AM, Lee ES, Granick MS. Interpersonal Violence as a Mechanisms of Injury. J Craniofac Surg 2015; 26 (5): 1446-1449.

13. Chao MT, Losee JE. Complications in Pediatric Facial Fractures. Craniomaxillofac Trauma Reconstr 2009; 2 (2): 103-112.

14. Iatrou I, Theologie-Lygidakis NT, Tzerbos F. Surgical protocols and outcome for the treatment of maxillofacial fractures in children: 9 years's experience. J Cranio Maxillofac Surg 2010; 38: 511-516.

15. Jingang A, Pengcheng J, Zhang Y, Gong X, Han X, He Y. Application of biodegradable plates for treating pediatric mandibular fractures. J Cranio-Maxillo-Fac Surg 2015; 43 (4): 515-520.

16. Kim YK, Kim SG. Treatment of mandible fractures using bioresorbable plates. Plast ReconstrS urg 2002; 110: 25-35.

17. Koltai PJ, Rabkin D, Hoehn J. Rigid fixation of facial fractures in children. J Craniomaxillofac Trauma 1995; 1: 32-42.

18. Koltai PJ, Rabkin D. Management of facial trauma in children. Pediatr Clin North Am 1996; 43: 1253-1275.

19. Lustmann J, Milhelm I. Mandibular fractures in infants. Review of the literature and report of seven cases. J Oral Maxillofac Surg 1994; 52: 240-246.

20. Neelam NA, Smriti CH, Ganapathy S. An institutional experience in the management of pediatric mandibular fractures: A study of 74 cases. J Cranio-Maxillo-Faci Surg 2015; 43: 995-999.

21. Posnick JC, Wells M, Pron G. Pediatric facial fractures: evolving patterns of treatment. J Oral MaxillofacSurg 1993; 51: 836-844.

22. Senel FC, Tekin US, Imamoglu M. Treatment of a mandibular fracture with biodegradable plate in an infant: Report of a case. Oral Surg Oral Med Oral Pathol Oral Radiol Andod 2006;101: 448-450.

23. Singhal R, Singh V, Bhagol A, Agrawal A, Kumar P. Pediatric maxillofacial injuries-if a new look is required? Int J Ped Otorhino 2013;77: 1333-1336.

24. Stanton DC, Liu F, Yu JW, Mistretta MC. Use of bioresorbable plating systems in paediatric mandible fractures. J Cranio-Maxillo-Fac Surg 2014; 42 (7): 1305-1309.

25. Starkhammar H, Olofsson J. Facial fractures: a review of 922 cases with special reference to incidence and aetiology. Clin Otolaryngol 1982; 7: 405 . 
26. Suuronen R, Haers PE, Lindqvist C, Sailer HF. Update on bioresorbable plates in maxillofacial surgery. Fac Plast Surg 1999; 15: 61-72.

27. Väänänen P, Nurmi JT, Nuutinen JP, Jakonen S, Happonen H, Jank S. Fixation properties of a biodegradable „free-form“ osteosynthesis plate. Oral Surg Oral Med Oral Pathol Oral Radiol Endod 2008; 106: 477-482.

28. Wheeler J, Phillips J. Pediatric Facial Fractures and Potential LongTerm Growth Disturbances. Craniomaxillofac Trauma Reconstr 2011; 4 (1): 43-52.

29. Yerit KC, Enislidis G, Schooper C, Turhani D, Wanschitz F, Wagner $\mathbf{A}$ et al. Fixation of mandibular fractures with biodgradable plates and screw. Oral Surg Oral Med Oral Pathol Oral Radiol Endod 2002; 94: 294-300.
30. Yerit KC, Hainich S, Enislidis G, Turhani D, Klug C, Wittwer G et al. Biodegradable fixation of mandibular fractures in children: stability and early results. Oral Surg Oral Med Oral Pathol Oral Radiol Endod 2005; 100: 17-24.

31. Zhi L, Ongodia D, Zu-Bing L. The use of resorbable plates in association with dental arch stabilization in the treatment of mandibular fractures in children. J Cranio-Maxillo-Fac Surg 2014; 42 (5): 548-551.

Received April 23, 2018. Accepted May 5, 2018. 